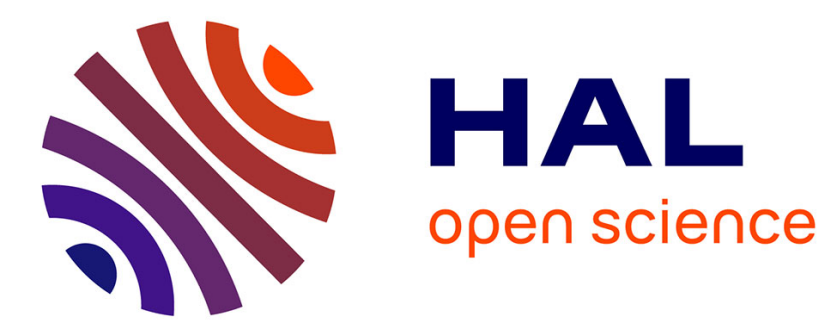

\title{
Développement et évaluation d'un modèle hydrologique distribué pour des bassins périurbains. Application au Bassin de l'Yzeron (150 km2)
}

M. Labbas, F. Branger, Isabelle Braud

\section{- To cite this version:}

M. Labbas, F. Branger, Isabelle Braud. Développement et évaluation d'un modèle hydrologique distribué pour des bassins périurbains. Application au Bassin de l'Yzeron (150 km2). La Houille Blanche - Revue internationale de l'eau, 2015, 5 (5), pp.84-91. 10.1051/lhb/20150059 . hal-01274928

\author{
HAL Id: hal-01274928 \\ https://hal.science/hal-01274928
}

Submitted on 22 Feb 2016

HAL is a multi-disciplinary open access archive for the deposit and dissemination of scientific research documents, whether they are published or not. The documents may come from teaching and research institutions in France or abroad, or from public or private research centers.
L'archive ouverte pluridisciplinaire HAL, est destinée au dépôt et à la diffusion de documents scientifiques de niveau recherche, publiés ou non, émanant des établissements d'enseignement et de recherche français ou étrangers, des laboratoires publics ou privés. 


\title{
Développement et évaluation d'un modèle hydrologique distribué pour des bassins périurbains - Application au bassin de l'Yzeron $\left(150 \mathrm{~km}^{2}\right)$
}

\author{
Mériem LABBAS ${ }^{1,2}$, Flora BRANGER ${ }^{1}$, Isabelle BRAUD ${ }^{1}$ \\ 1. Irstea, UR HHLY, centre de Lyon-Villeurbanne, 5 rue de la Doua CS70077 69626 Villeurbanne Cedex - e-mail: meriem.labbas@gmail.com \\ 2. AgroParisTech, F-75005 Paris, France
}

\begin{abstract}
RÉSUMÉ. - L'augmentation des surfaces imperméables et les modifications des chemins d'écoulement de l'eau par les réseaux d'assainissement unitaires et pluviaux ont un impact sur l'hydrologie des bassins versants. Les bassins périurbains, qui s'urbanisent rapidement, y sont particulièrement sensibles. Les outils de modélisation hydrologique spatialisés, simulant l'ensemble du cycle hydrologique et capables de prendre en compte l'hétérogénéité très importante de ces bassins, sont utiles à l'évaluation de l'influence des modes de gestion des eaux pluviales sur l'hydrologie. Nous proposons un nouvel outil de modélisation adapté à ces problématiques : le modèle distribué horaire J2000P. Ce modèle simule les processus hydrologiques en milieux ruraux et urbains et prend en compte les réseaux d'assainissement, les connexions à ces réseaux et les déversements des déversoirs d'orage (DO). J2000P a été mis en œuvre sur le bassin périurbain de l'Yzeron $\left(\sim 150 \mathrm{~km}^{2}\right)$, situé à l'ouest de Lyon. L'évaluation, effectuée à l'exutoire de différents sous-bassins de tailles et d'occupations du sol différentes, montre des résultats très encourageants. Le modèle a tendance à sous-estimer le débit mais la dynamique des pics est bien représentée tout comme le déversement des DO.
\end{abstract}

Mots-clés : déversoir d'orage, JAMS, J2000P, modélisation hydrologique distribuée, réseau unitaire.

\section{Development and evaluation of a periurban distributed hydrological model - Application to the Yzeron catchment $\left(150 \mathrm{~km}^{2}\right)$}

\begin{abstract}
Growing urbanization and related anthropogenic processes have a high potential to influence hydrological process dynamics. Periurban catchments, at the edge of large cities, are especially affected by fast anthropogenic modifications. Spatialized hydrological modeling tools, simulating the entire hydrological cycle and able to take into account the important heterogeneity of periurban watersheds can be used to assess the impact of stormwater management practices on their hydrology. We propose a new modeling tool for these issues: the hourly distributed J2000P model. This model simulates the hydrological processes in rural and urban areas and takes into account the sewerage networks, connections to these networks and overflows from sewer overflow devices (SOD). The application site is the Yzeron catchment $\left(\sim 130 \mathrm{~km}^{2}\right)$, located in the West of Lyon. The evaluation, conducted at the outlet of different sub-basins with different sizes and land use, shows very encouraging results. The model tends to underestimate the discharge but the dynamics of the peaks and the SOD overflows are well simulated.
\end{abstract}

Key-words: sewer overflow device, JAMS, J2000P, distributed hydrological modelling, sewer network.

\section{INTRODUCTION}

Les bassins périurbains, constitués d'un «patchwork » de zones urbaines, agricoles et " naturelles » [Andrieu et Chocat, 2004] sont des bassins versants complexes à étudier. L'augmentation des surfaces imperméables et les modifications des chemins d'écoulement par les réseaux d'assainissement influencent leur bilan hydrique et leur temps de réponse. Ces modifications sont notamment liées aux choix des modes de gestion des eaux pluviales : réseaux unitaires ou séparatifs, infiltration à la parcelle, etc. Le transport des eaux usées et pluviales à la station d'épuration (STEP) ou à la rivière, parfois en dehors du bassin versant, modifie les frontières hydrologiques du bassin. Les déversements des DO peuvent influencer le régime des rivières, en plus de diminuer la qualité de l'eau. Braud et al. [2013a] ont montré que les DO peuvent devenir les principaux contributeurs de petites rivières en période sèche. Les processus hydrologiques souterrains sont modifiés par la présence des réseaux qui peuvent drainer l'eau des nappes quand leur niveau est suffisamment haut [Rodriguez et al., 2008]. La modélisation hydrologique spatialisée est un outil permettant d'étudier et de quantifier les impacts de l'urbanisation croissante sur le régime hydrologique. Peu de modèles ont été développés pour être appliqués aux bassins périurbains, à l'échelle des gestionnaires et pour des simulations sur de longues périodes (>10 ans). Cet article décrit le développement d'un modèle hydrologique distribué J2000P adapté aux bassins périurbains de taille intermédiaire $\left(100-500 \mathrm{~km}^{2}\right)$. Une fois validé, ce modèle sera utilisé comme un outil permettant de tester différentes hypothèses de fonctionnement afin d'améliorer la compréhension des processus en milieu périurbain.

La modélisation hydrologique de bassins périurbains est assez récente. Andrieu et Chocat [2004] avaient souligné 
qu'il était temps de développer des modèles pour le milieu périurbain et depuis, les recherches ont avancé sur ce domaine [Braud et al., 2013b]. Usuellement, les modèles sont différenciés selon le milieu étudié : rural ou urbain. Avec les modèles dits « urbains », comme CANOE [Insavalor et Sogreah, 1997] ou MOUSE [DHI, 2011], les objectifs poursuivis sont généralement le dimensionnement et la compréhension du fonctionnement des réseaux d'assainissement. D'autres décrivent à la fois la génération des écoulements et leurs transferts. C'est le cas d'URBS [Rodriguez et al., 2008], mis en œuvre sur des petits bassins versants. Dans les modèles ruraux, l'objectif est de représenter au mieux les processus hydrologiques pour simuler la génération des écoulements. Le réseau d'assainissement n'y est pas représenté. Peu à peu, ces modèles ont été adaptés au milieu périurbain. C'est le cas de la version périurbaine d'ISBA-TOPMODEL [Furusho et al., 2013] qui décrit le ruissellement sur surfaces imperméables et l'infiltration des eaux souterraines dans le réseau. Dans le modèle MERCEDES [Lhomme et al., 2004], le routage dans le réseau d'assainissement est pris en compte en diminuant l'altitude des cellules où se trouvent le réseau. ArcEGMO-Urban [Biegel et al., 2005], basé sur un modèle hydrologique rural, intègre des données urbaines pour modéliser le réseau d'assainissement et les flux de pollution. D'autres modèles dits « mixtes» combinent les deux approches. Par exemple, l'outil de modélisation MIKE [Gustafsson, 2000] inclut le modèle distribué MIKE-SHE, le modèle de réseau MOUSE et le modèle hydraulique MIKE11 ou MIKE 21 simulant les écoulements 1D ou 2D. Le modèle BlueM.Sim [Bach et al., 2010] utilise une plateforme de modélisation pour coupler un modèle urbain, un modèle rural et un modèle de routage pour simuler les flux de polluants. Le modèle PUMMA [Jankowfsky et al., 2014], déjà mis en œuvre sur un petit sous-bassin de l'Yzeron $\left(2,7 \mathrm{~km}^{2}\right)$, se base sur la combinaison de modèles ruraux et urbains réunis au sein d'une plateforme de modélisation. L'approche orientée objet utilisée contraint cependant la mise en œuvre du modèle sur des bassins de plus grande taille. La modélisation hydrologique doit donc encore évoluer afin de mieux capter les spécificités des bassins périurbains de taille intermédiaire et simuler les processus sur le long terme. C'est ce qui a motivé les travaux dont nous rendons compte ici.

\section{MÉTHODOLOGIE}

\section{II.1. Présentation du bassin versant étudié}

Le bassin de l'Yzeron, d'une superficie de $147 \mathrm{~km}^{2}$, s'étend de la vallée de l'Yzeron jusqu'aux Monts du Lyonnais où il culmine à $912 \mathrm{~m}$ (Figure 1). L'exutoire du bassin se situe à $162 \mathrm{~m}$ d'altitude à la hauteur de la ville d'Oullins où la rivière Yzeron se jette dans le Rhône. C'est un bassin représentatif du milieu périurbain avec une occupation du sol très hétérogène [Jacqueminet et al., 2013] En amont, les sols pentus sont recouverts de forêts et de parcelles agricoles. Le bassin s'urbanise ensuite de plus en plus jusqu'à l'aval où se trouve l'agglomération lyonnaise. Le socle géologique se compose essentiellement de gneiss et de granite, avec quelques formations alluvionnaires à l'aval. Les sols, peu profonds et peu perméables, conduisent à une faible capacité de stockage. Le régime de type pluvial est contrasté avec de fortes périodes d'étiage en été et des crues importantes et rapides au printemps et en automne. La pluie moyenne annuelle est de $830 \mathrm{~mm}$. Le climat combiné à la topographie et à la pédologie conduit à des inondations rapides dans la partie la plus urbanisée en aval. Le temps de réponse du bassin a été estimé à 12h [Braud et al., 2013a]. Depuis les années 1970, ce bassin est soumis à une forte augmentation de l'urbanisation. Malgré une politique active de déconnexion des eaux pluviales menée depuis les années 2000 par le Grand Lyon, la gestion des eaux pluviales se fait encore majoritairement au moyen de réseaux unitaires (Figure 1). Des déversoirs d'orage sont présents le long du réseau pour limiter la saturation de la STEP en cas de fortes pluies. La quasi-totalité des communes du bassin est raccordée au réseau d'assainissement du Grand Lyon. Celui-ci

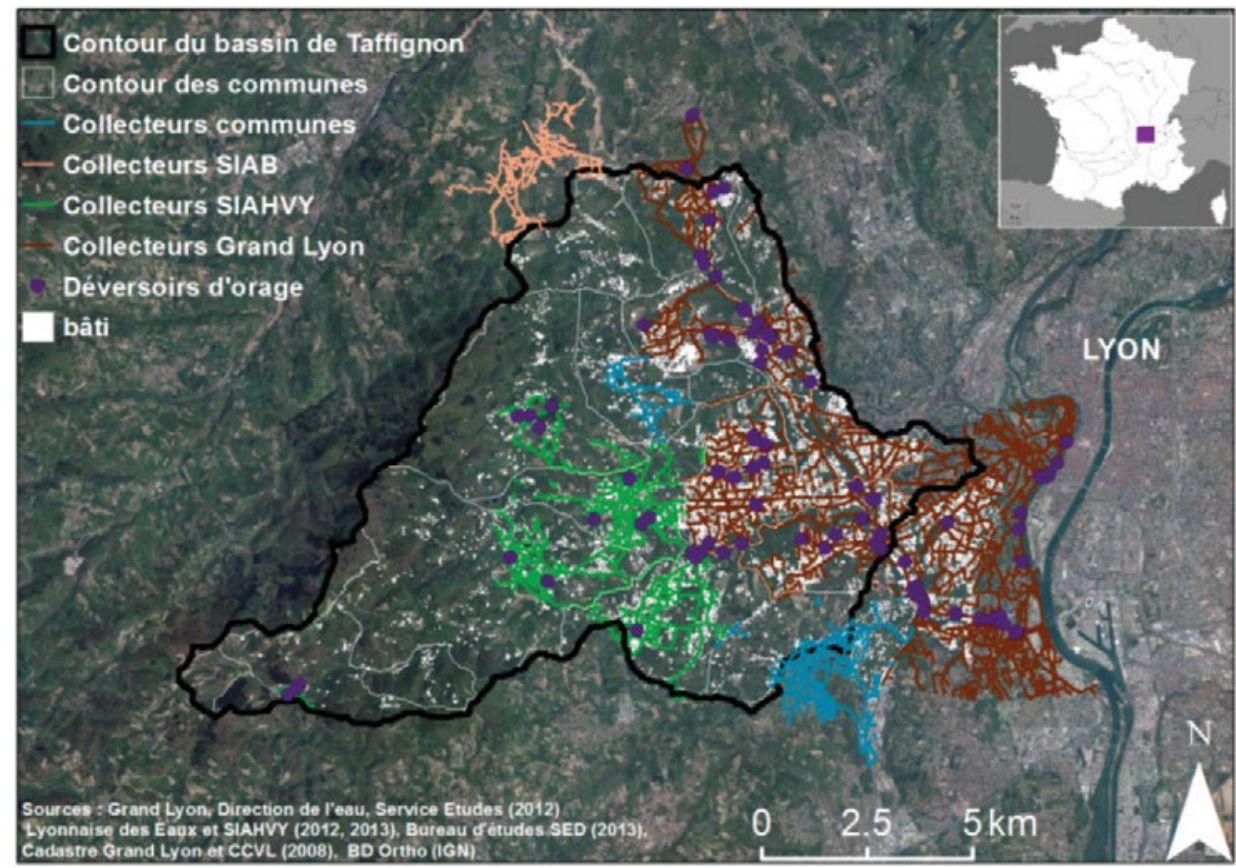

Figure 1 : Le bassin versant de l'Yzeron et ses réseaux d'assainissement collectif (collecteurs et DO), gérés par différentes entités. 
rejoint la station de Pierre-Bénite qui rejette dans le Rhône en dehors du bassin. Les dysfonctionnements du réseau d'assainissement sont nombreux : mauvais branchements, eaux claires parasites permanentes dues aux branchements de sources sur le réseau ou à des infiltrations de l'eau de nappe.

\section{II.2. Données disponibles}

Le bassin de 1'Yzeron est instrumenté depuis les années 1960. Il fait partie de l'Observatoire de Terrain en Hydrologie Urbaine (OTHU), mis en place en 1999 pour étudier les rejets urbains de temps de pluie. Les débits sont mesurés par la DREAL Rhône-Alpes et Irstea à l'exutoire de 7 sous-bassins, pour certains emboîtés (Figure 2). L'ensemble des caractéristiques liées à ces stations de mesure est résumé dans le Tableau 1. Cinq DO sont instrumentés par le Syndicat d'Assainissement de la Haute Vallée de l'Yzeron (SIAHVY) dans le cadre de l'auto-surveillance depuis 2010 et un DO par Irstea depuis 2001. Plusieurs stations pluviométriques, suivies par le Grand Lyon, Irstea et Météo-France depuis 2 à 25 ans, sont réparties sur le bassin (Figure 2). Les données climatiques nécessaires au calcul de l'évapotranspiration de référence $\left(E T_{r e f}\right)$ sont issues de la réanalyse SAFRAN disponible sur toute la France au pas de temps horaire sur une grille de $8 \times 8 \mathrm{~km}^{2}$ [Vidal et al., 2010]. Les données géographiques sont : un MNT issu de la BD TOPO de l'IGN à la résolution de $25 \mathrm{~m}$, une carte pédologique du programme $\mathrm{DONESOL}^{1}$ une carte géologique digitalisée à partir de cartes papier du BRGM au 1:50 000. La donnée d'occupation du sol résulte de la synthèse à $2,5 \mathrm{~m}$ de plusieurs sources d'imagerie à haute résolution pour l'année 2008 [Jacqueminet et al., 2013]. Le réseau hydrographique

1. http://www.gissol.fr/outil/donesol/donesol.php

Tableau 1 : Description des stations hydrométriques du bassin de l'Yzeron. Les zones rurales désignent des zones faiblement urbanisées, principalement recouvertes de forêts et de cultures.

\begin{tabular}{|c|c|c|c|c|c|c|c|}
\hline \multirow[t]{2}{*}{ Nom Station } & \multirow{2}{*}{$\begin{array}{c}\text { Surface } \\
\text { drainée } \\
\left(\mathbf{k m}^{2}\right)\end{array}$} & \multicolumn{2}{|c|}{$\begin{array}{c}\text { Occupation des sols } \\
\text { dominante }\end{array}$} & \multirow{2}{*}{$\begin{array}{c}\text { Période } \\
\text { disponible }\end{array}$} & \multirow{2}{*}{$\begin{array}{l}\text { Gestion- } \\
\text { naire }\end{array}$} & \multicolumn{2}{|c|}{$\begin{array}{l}\% \text { de surfaces } \\
\text { imperméables }\end{array}$} \\
\hline & & amont & aval & & & total & connectées \\
\hline Taffignon & 129 & rural & très urbain & 1988-2012 & DREAL & 16,4 & 8,7 \\
\hline Craponne & 48 & rural & urbain & $1968-2012$ & DREAL & 10,7 & 4,8 \\
\hline Charbonnières & 22,9 & rural & urbain & 2010-2012 & Irstea & 11,3 & 4,1 \\
\hline Ratier & 18,9 & rural & urbain & 2009-2012 & Irstea & 9,1 & 4,29 \\
\hline Mercier & 6,7 & forêt & cultures & $1997-2012$ & Irstea & 5,5 & 1,8 \\
\hline La Léchère & 4,2 & cultures & urbain & 2005-2012 & Irstea & 19,6 & 11,8 \\
\hline PontBarge & 2,2 & cultures & urbain & $\begin{array}{l}1997-2001 \\
2005-2012\end{array}$ & Irstea & 15,8 & 8,6 \\
\hline
\end{tabular}

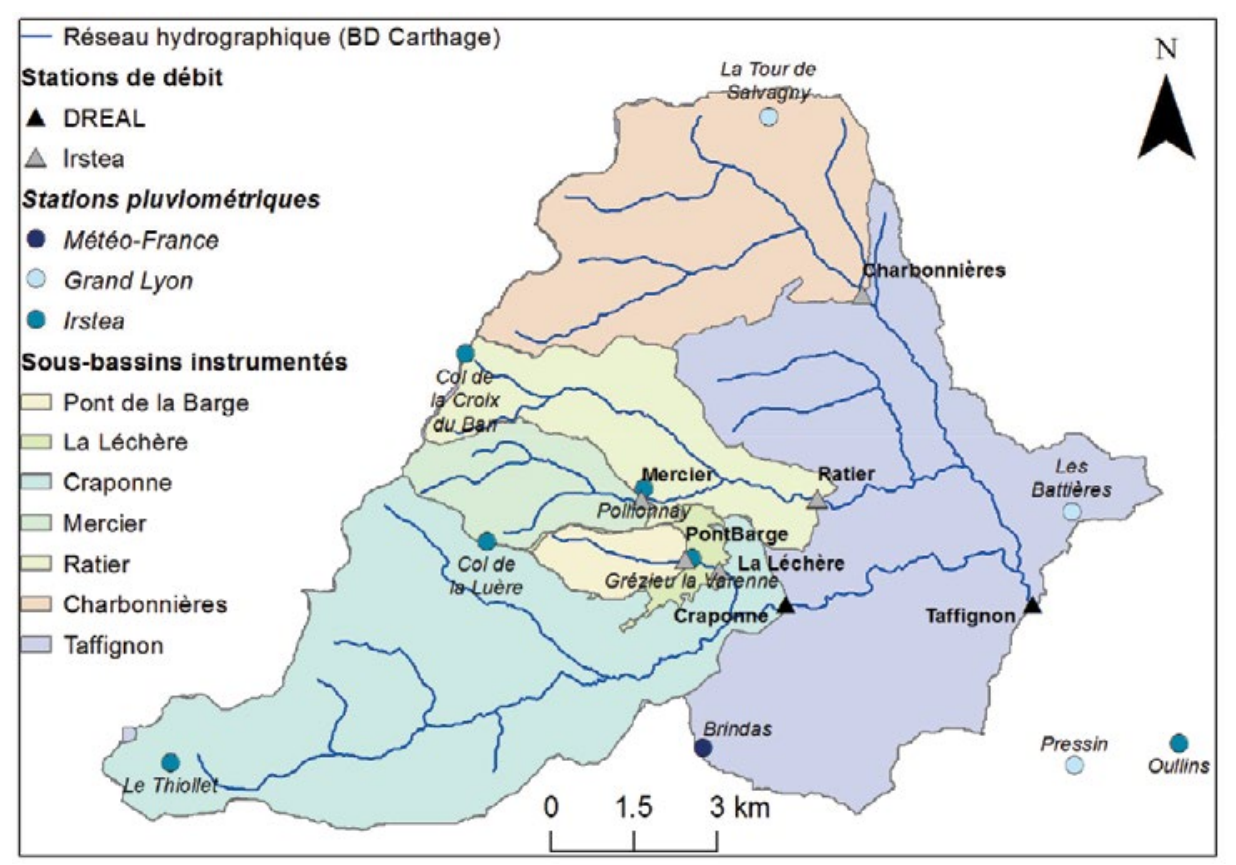

Figure 2 : Emplacement des stations débitmétriques et pluviométriques et cartographie des sous-bassins drainés par les stations de débits. 
provient de la BD Carthage complétée par des mesures de terrain [Schmitt et al., 2004]. Les données sur les réseaux ont été récupérées auprès du Grand Lyon, de syndicats d'assainissement et de communes.

\section{II.3. Développement d'un outil de modélisation : le modèle $\mathrm{J2000P}$}

L'outil de modélisation développé est le modèle distribué multi-exutoire J2000P (J2000 Periurban) [Labbas et al., 2013] qui fonctionne à partir de la plateforme de modélisation JAMS (Jena Adaptable Modelling System, Kralisch et al., 2007], sur la base du modèle journalier simplifié à base physique J2000 [Krause et al., 2006 ; Branger et al., 2013]. L'originalité du fonctionnement modulaire de la plateforme de modélisation et la disponibilité des codes en open source ont conditionné le choix du modèle ${ }^{2}$.

De manière à mieux prendre en compte la dynamique rapide des processus, J2000P fonctionne au pas de temps horaire plutôt que journalier. La discrétisation spatiale est faite sur un maillage irrégulier, en unités de réponse hydrologique (HRU) [Flügel, 1995]. A chaque pas de temps, le modèle simule le comportement hydrologique de chaque HRU en réponse aux forçages et calcule leur bilan hydrique. La représentation des processus hydrologiques s'appuie sur une approche par réservoirs, caractérisés par des paramètres à base physique ${ }^{3}$. Les principaux processus représentés sont : l'évapotranspiration, l'interception, le ruissellement direct sur les surfaces imperméables (RD1), l'infiltration, l'écoulement de subsurface (RD2), le stockage dans le sol et dans la nappe souterraine, et le débit de base (RG1) (Figure 3). Dans J2000P, la partition entre le ruissellement direct et l'infiltration a été modifiée pour être contrôlée par un paramètre distribué $\beta_{\text {inf }}$ ou coefficient d'infiltration, propre à chaque

2. JAMS et le modèle de base J2000 sont téléchargeables gratuitement ici ; http://jams.uni-jena.de/.

3. http://jams.uni-jena.de/ilmswiki/index.php/Hydrological_Model_J2000
HRU. Le débit total est calculé à partir de la production et du transfert dans les unités de modélisation de trois flux différents : RD1, RD2 et RG1.

Le routage s'effectue dans le réseau hydrographique constitué de brins de rivière et dans un réseau d'assainissement explicitement décrit découpé en tronçons (Figure 3). Pour le moment, seul le ruissellement direct peut être transféré à la fois au réseau hydrographique et au réseau d'assainissement. La répartition entre les deux réseaux est contrôlée par le paramètre distribué $\tau_{\text {conec }}$, qui détermine le taux de connexion au réseau de chaque HRU. De plus, un module DO a été développé pour simuler les déversements du réseau dans la rivière quand le niveau d'eau dans le réseau atteint un certain seuil (Figure 3).

\section{APPLICATION DE J2000P AU BASSIN DE L'YZERON}

\section{III.1. Construction d'un maillage adapté}

Le maillage en HRU choisi est constitué de sousbassins versants, catégorisés en " ruraux » ou en " mixtes » (Figure 4). Dans les sous-bassins dits ruraux, le ruissellement produit par les zones urbaines rejoint directement le brin de rivière drainant le sous-bassin. La gestion des eaux pluviales dans les zones urbaines de ces sousbassins se fait à la source ou par des réseaux séparatifs. Ces réseaux ne sont pas explicitement décrits car nous faisons l'hypothèse qu'ils sont peu nombreux et qu'ils sont connectés à la rivière à l'intérieur du sous-bassin. Dans les sousbassins dits mixtes, une partie du ruissellement des eaux pluviales généré par les zones imperméables est transférée au réseau unitaire, explicitement décrit et le reste est transféré au réseau hydrographique.

Les sous-bassins ruraux et mixtes sont issus de l'intersection de sous-bassins naturels et urbains. Les sous-bassins naturels ont été délimités automatiquement à partir

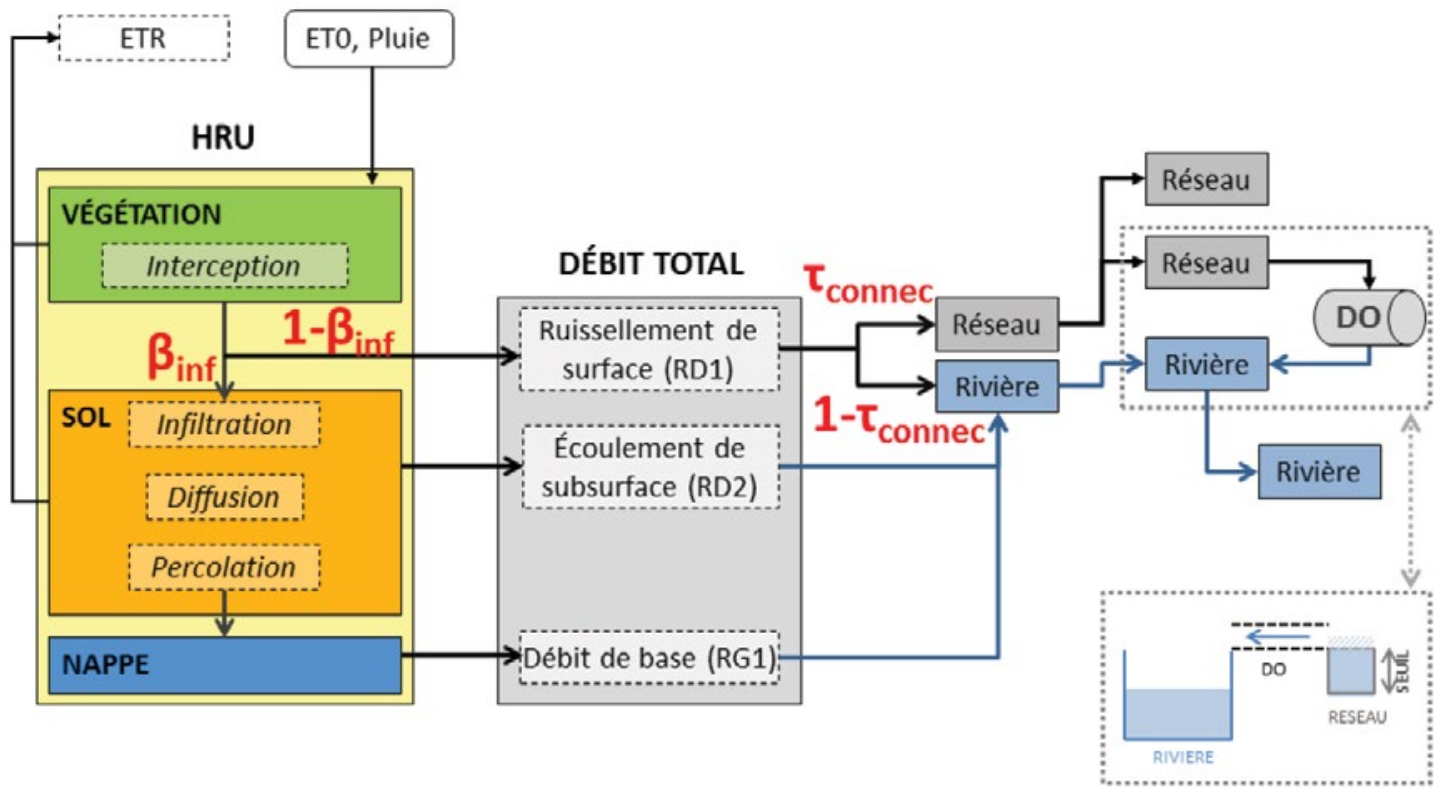

Figure 3 : Processus hydrologiques se produisant dans une HRU. La répartition du ruissellement direct entre les réseaux hydrographique et unitaire est contrôlée par le paramètre $\tau$ d'eau dépasse un certain seuil. Des DO déversent l'eau du réseau dans la rivière lorsque la hauteur 


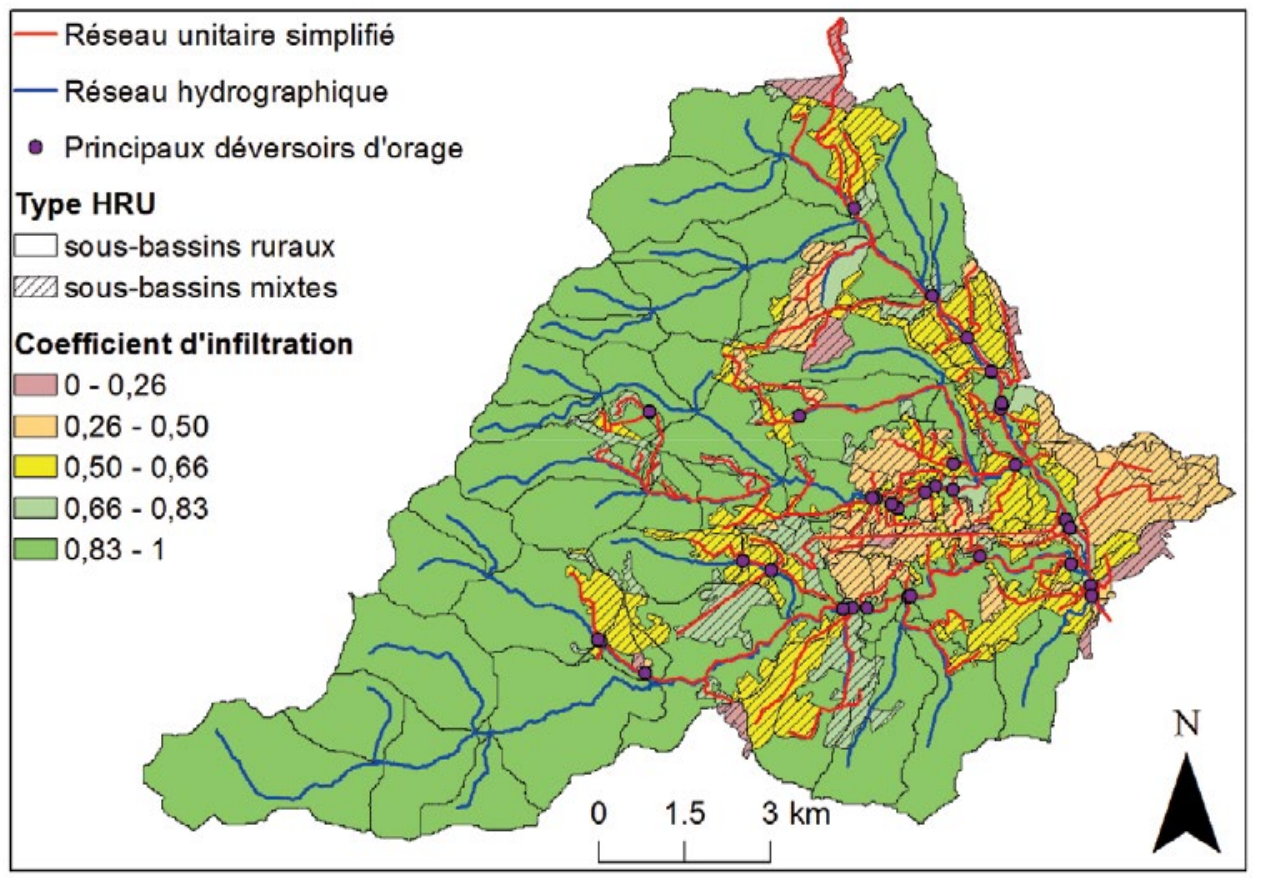

Figure 4 : Maillage en sous-bassins et coefficients d'infiltration rattachés.

de la topographie et du réseau hydrographique. Les sousbassins urbains ont été délimités manuellement à partir des données sur les réseaux et leur sens d'écoulement, les zonages d'assainissement, le cadastre et l'expertise de terrain. Ils sont issus des travaux d'AVuPUR [Braud et al., 2010] pour la partie gérée par le SIAHVY à l'ouest, des travaux du Grand Lyon réalisés dans le cadre de l'application du modèle CANOE au bassin de l'Yzeron pour la partie est du bassin et de nouveaux découpages dans les zones où de nouvelles données ont été récupérées. Le bassin est ainsi composé de 310 HRU : 98 sous-bassins ruraux $\left(\right.$ Stot $=94 \mathrm{~km}^{2}$, Smoy $\left.=0,9 \mathrm{~km}^{2}\right)$ et 212 sous-bassins mixtes $\left(\right.$ Stot $=35 \mathrm{~km}^{2}$, Smoy $\left.=0,16 \mathrm{~km}^{2}\right)$. Le réseau hydrographique est constitué de 85 brins de longueur moyenne $1.3 \mathrm{~km}$. Le réseau unitaire modélisé a été sélectionné à partir des collecteurs de plus grands diamètres : 185 tronçons de longueur moyenne $700 \mathrm{~m}$ (Figure 4). $34 \mathrm{DO}$ ont été identifiés à partir de travaux réalisés dans le cadre du projet AVuPUR.

\section{III.2. Forçages et paramétrage}

Une $E T_{\text {ref }}$ uniforme sur le bassin a été calculée en dehors du modèle à partir des variables climatiques des 7 mailles SAFRAN qui intersectent le bassin, selon la méthode FAO [1998]. La pluie de chaque maille est calculée par un module de régionalisation à partir des 4 pluviomètres les plus proches par la méthode inverse des distances. La spécification des paramètres du modèle est effectuée sans calage, à partir des données disponibles ou de valeurs trouvées dans la littérature. Pour simplifier le paramétrage, des classes de végétation, sol et sous-sol ont été définies pour attribuer les paramètres de chaque HRU en fonction du type de végétation, sol et sous-sol majoritaire. La végétation a été catégorisée en trois classes : culture, forêt et urbain. Pour chacune de ces classes, des valeurs moyennes d'indice foliaire, coefficient cultural et profondeur racinaire ont été calculées à partir des bases FAO [1998] et Ecoclimap [Masson et al., 2003]. Cinq classes de sol ont été définies et attribuent à chaque HRU une épaisseur moyenne du sol (50 à $200 \mathrm{~cm}$ ), une capacité d'air (175 à $650 \mathrm{~mm}$ ) et une capacité au champ $(225$ à $800 \mathrm{~mm})$. La base de données DONESOL ne décrivant pas les sols urbains, une reconstitution des sols a été effectuée à partir de la cartographie des sols hors urbains, du réseau hydrographique et de la géologie. Deux classes de sous-sol décrivent la taille des réservoirs de nappe et leur taux de vidange $(10 \mathrm{j})$.

Le coefficient d'imperméabilisation spatialisé $\left(1-\beta_{\text {inf }}\right)$ est estimé à partir du pourcentage de zones imperméables dans chaque sous-bassin. Dans les sous-bassins ruraux, ce coefficient est en moyenne de 0,12 $(\sigma=0,14)$. Dans les sousbassins mixtes, il est en moyenne de 0,46 $(\sigma=0,17)$. Certains sous-bassins mixtes peuvent être faiblement imperméabilisés (sous-bassins verts hachurés) et vice et versa (Figure 4). La majorité du réseau d'assainissement du bassin collectant les eaux en milieu très urbanisé, le taux de connexion $\tau$ connec a été fixé à une seule valeur de $70 \%$. Cette valeur étant difficile à estimer, une analyse de sensibilité sur ce paramètre a été effectuée. Le paramétrage du réseau hydrographique et du réseau d'assainissement (pente, largeur, rugosité) s'est basé sur les données disponibles et les données manquantes ont été extrapolées. La rugosité a été évaluée à 25 pour la rivière et à 75 pour le réseau d'après les informations recueillies sur les matériaux. Pour les DO, une hauteur seuil de $40 \mathrm{~cm}$ a été choisie, ce qui correspond à la hauteur moyenne des DO répertoriés par le Grand Lyon sur le bassin de l'Yzeron.

\section{IV. ÉVALUATION DU MODÈLE ET DISCUSSION}

Les simulations sont effectuées sur la période 2003-2012 et le modèle est évalué sur la période 2005-2012 pour correspondre aux données d'occupation du sol (carte de 2008). Pour cette période, la pluie moyenne annuelle du bassin est de $762 \mathrm{~mm}$. 
Les premiers résultats sont encourageants comme le confirme l'analyse de différents critères de performance du modèle, à l'exutoire des 7 sous-bassins instrumentés (Tableau 2). Le critère de biais montre que le modèle a tendance à sous-estimer les débits. D'après les valeurs du critère de Nash classique $(\mathrm{Q})$, la dynamique des pics de débit est bien modélisée sauf à l'exutoire des deux sous-bassins du Charbonnières et du Ratier. Les critères sont meilleurs au pas de temps agrégé journalier qu'au pas de temps horaire, ce qui montre la capacité du modèle à étudier les variations du débit sur de longues périodes plutôt que sur un évènement particulier. Le critère de Nash calculé à partir de la racine carrée des débits est moins bon et souligne la difficulté à simuler la dynamique des débits moyens. Ceci est visible également sur la Figure 5 où sont comparés les débits simulés et observés, agrégés au pas de temps journalier, à l'exutoire du bassin de Taffignon pour l'année 2008. La représentation avec l'échelle logarithmique souligne que le modèle rend bien compte de la dynamique des pics de débit mais qu'au début du printemps et en été, les récessions sont trop rapides et les bas débits trop faibles. Ce phénomène est confirmé par la comparaison des bas débits observés et simulés (Tableau 2) : simulations et observations sont en bon accord pour 4 sous-bassins dont le bassin de Taffignon et pas pour les sous-bassins de Craponne, Charbonnières et Ratier. Les débits moyens simulés sont toujours inférieurs aux débits moyens observés. A l'opposé, la comparaison des débits maximaux montre que le modèle a plutôt tendance à surestimer les pics de crue, notamment pour le bassin de Taffignon. Ces résultats soulignent la réactivité trop

Tableau 2: Critères de performance calculés pour l'évaluation du modèle. Les critères de Nash ont été calculés au pas de temps horaire (en gras) et agrégé journalier. Le seuil choisi pour déterminer le pourcentage de bas débits de chaque sous-bassin est $10^{-3}$ fois le débit moyen observé du sous-bassin. Les débits moyens et maximaux sont ceux obtenus au pas de temps horaire.

\begin{tabular}{|c|c|c|c|c|c|c|c|c|c|}
\hline Bassin & $\operatorname{Nash}(Q)(-)$ & $\operatorname{Nash}(\sqrt{ } Q)(-)$ & $\begin{array}{c}\text { Biais } \\
(\%)\end{array}$ & $\begin{array}{c}\text { Bas débits } \\
\text { Sim (\%) }\end{array}$ & $\begin{array}{c}\text { Bas } \\
\text { débits } \\
\text { Obs }(\%)\end{array}$ & $\begin{array}{c}Q_{\text {mean }}^{\text {sim }} \\
\left(\mathbf{m}^{3} \mathbf{s}^{-1}\right)\end{array}$ & $\begin{array}{c}Q_{\text {mean }}^{\text {obs }} \\
\left(\mathbf{m}^{3} \mathbf{s}^{-1}\right)\end{array}$ & $\begin{array}{c}Q_{\max }^{s i m} \\
\left(\mathbf{m}^{3} \mathbf{s}^{-1}\right)\end{array}$ & $\begin{array}{c}Q_{\max }^{o b s} \\
\left(\mathrm{~m}^{3} \mathbf{s}^{-1}\right)\end{array}$ \\
\hline Taff. & $\mathbf{0 , 4 6} / 0,77$ & $\mathbf{0 , 5 6} / 0,65$ & -5 & 5,2 & 2,0 & 0,589 & 0,625 & 136,8 & 68,01 \\
\hline Crap. & $\mathbf{0 , 6 5} / 0,72$ & $\mathbf{0 , 3 4} / 0,39$ & -38 & 12,5 & 0 & 0,195 & 0,315 & 52,31 & 41,34 \\
\hline Charbo. & $\mathbf{0 , 3 4} / 0,44$ & $\mathbf{0 , 0 1} / 0,03$ & -43 & 12,0 & 0 & 0,056 & 0,098 & 6,23 & 6,41 \\
\hline Ratier & $\mathbf{0 , 2 9} / 0,41$ & $\mathbf{0 , 4 7} / 0,51$ & -17 & 10,1 & 0,1 & 0,084 & 0,102 & 5,32 & 2,14 \\
\hline Merc. & $\mathbf{0 , 6 4 / 0 , 6 5}$ & $\mathbf{0 , 4 9} / 0,50$ & -24 & 15 & 12 & 0,034 & 0,049 & 8,75 & 9,50 \\
\hline Léch. & $\mathbf{0 , 6 3} / 0,68$ & $\mathbf{0 , 4 8} / 0,54$ & -34 & 14,6 & 12,9 & 0,012 & 0,018 & 2,17 & 2,88 \\
\hline PontB. & $\mathbf{0 , 4 6} / 0,60$ & $\mathbf{0 , 2 8} / 0,36$ & -7 & 16,1 & 35,7 & 0,011 & 0,011 & 1,73 & 2,85 \\
\hline
\end{tabular}
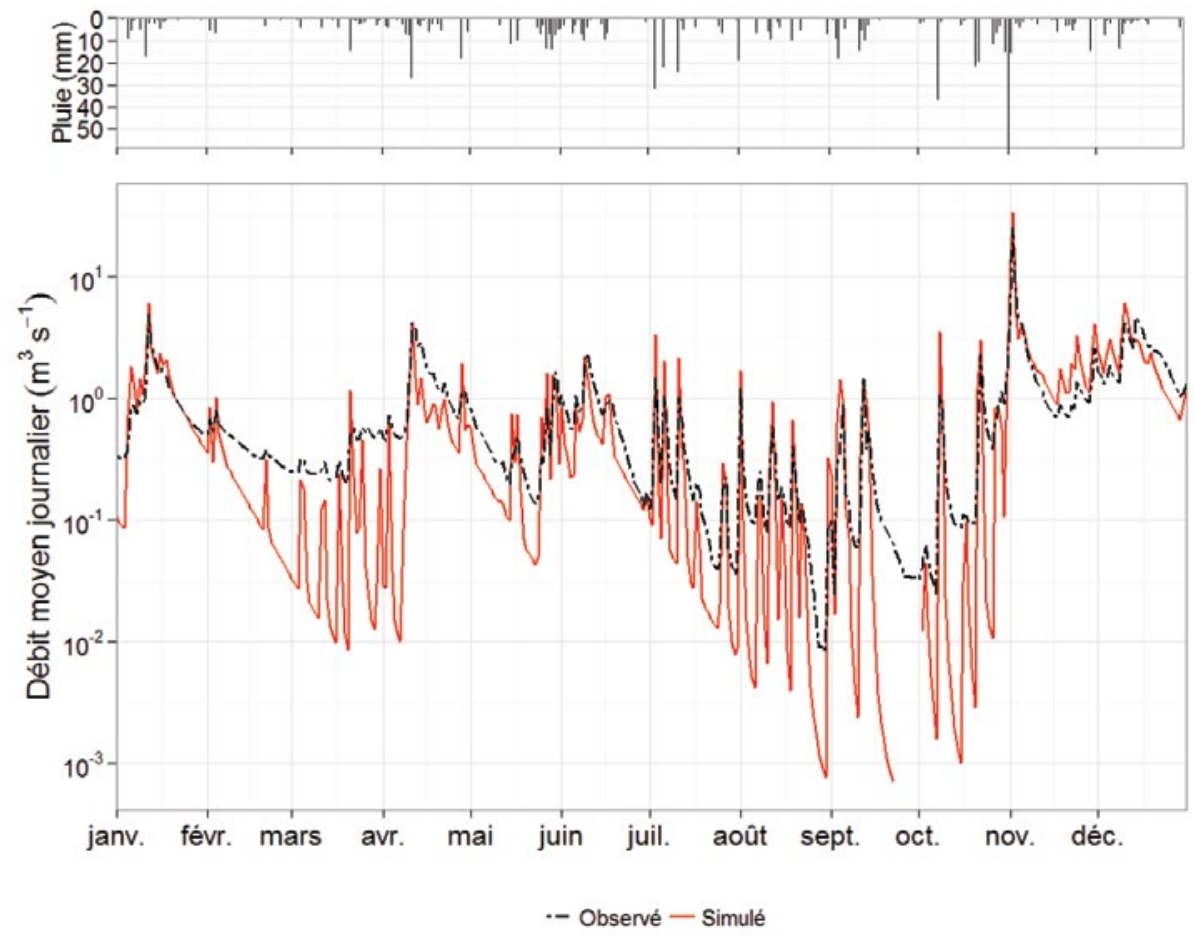

Figure 5 : Débit moyen journalier observé et simulé à l'exutoire de Taffignon (échelle log) et pluie journalière pour l'année 2008. 


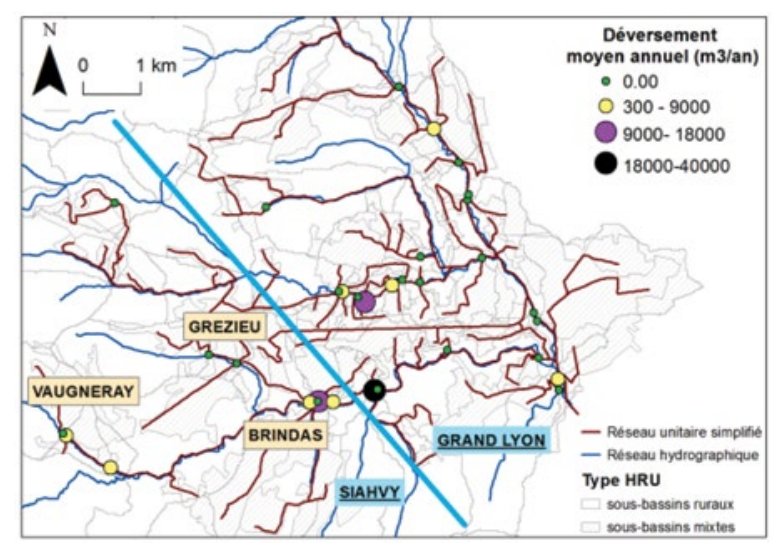

Figure 6 : Déversements moyens annuels des DO entre 2005 et 2012. Zoom sur l'aval urbanisé.

forte du modèle : pics importants et récessions trop rapides. La représentation du ruissellement sur les surfaces imperméables peut être à l'origine de ce comportement. A cette étape de l'évaluation du modèle, peu de différences sont observées entre les comportements des différents sous-bassins, qui ont pourtant des occupations du sol assez différentes. Pour la période estivale, où la contribution majeure au débit est le ruissellement direct, une analyse de sensibilité au taux de connexion a été menée (Figure 7). Une augmentation de $\pm 43 \%$ de ce taux entraine une variation de débit moyen interannuel de $0,05 \mathrm{~m}^{3} \mathrm{~s}^{-1}$ en période estivale $(7,5 \%$ du débit moyen interannuel observé).

Le modèle simule des déversements dans 11 DO : 5 dans la partie gérée par le SIAHVY, 6 dans la partie gérée par le Grand Lyon (Figure 6). Des analyses plus détaillées sont nécessaires mais les premiers résultats montrent qu'à l'ouest, dans la partie gérée par le SIAHVY, les déversements simulés correspondent géographiquement à ceux observés à Vaugneray et à Brindas, dans le cadre de l'autosurveillance des DO. Cependant, le modèle ne simule pas de déversements dans les 2 DO instrumentés par le SIAHVY et Irstea à Grézieu alors que des déversements y sont observés. Une première comparaison des résultats de simulation du modèle J2000P et du modèle CANOE dans la partie aval gérée par le Grand Lyon montre que les volumes moyens déversés simulés par J2000P sont inférieurs à ceux simulés par CANOE (résultats non représentés sur la figure). CANOE simule également des déversements à plus d'endroits que J2000. Le modèle simule donc bien des déversements mais pas autant en nombre et en quantité que dans la réalité ou que dans un modèle détaillant plus précisément les singularités du réseau. La tendance de J2000P à sous-estimer les débits et donc certainement le ruissellement peut être une cause de ce résultat.

\section{CONCLUSIONS ET PERSPECTIVES}

Par rapport aux travaux passés [Branger et al., 2013], les résultats obtenus avec le modèle $\mathrm{J} 2000 \mathrm{P}$ suite à la spatialisation du coefficient d'infiltration et à l'ajout du réseau d'assainissement sont très encourageants. Cela montre l'importance de la prise en compte des processus hydrologiques en milieu urbain pour étudier des bassins périurbains de taille intermédiaire. Des tests de sensibilité à certains paramètres tels que le coefficient de vidange du réservoir de

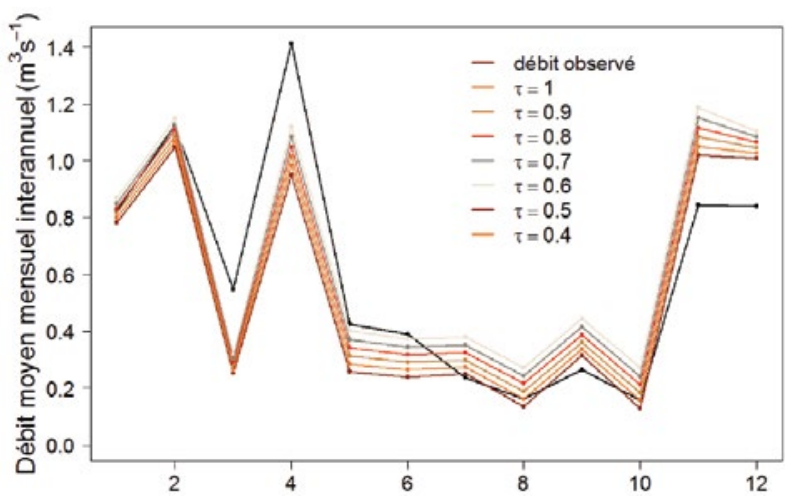

Figure 7 : Sensibilité du modèle au paramètre de taux de connexion: débits moyens mensuels interannuels simulés entre 2005 et 2010 pour différents taux de connexion.

nappe ou le coefficient de rugosité de la rivière devraient permettre d'identifier plus précisément les paramètres les plus sensibles et les processus mal représentés afin d'apporter des améliorations au modèle. Ces analyses de sensibilité permettront de tester des hypothèses de fonctionnement et de hiérarchiser l'importance des différents processus hydrologiques. La prise en compte des incertitudes sur les débits observés [Le Coz et al., 2013] et la pluie pourra également aider à mieux évaluer le modèle. Des cartes d'occupation du sol sont par ailleurs disponibles à plusieurs dates entre 1945 et 2008 et seront utilisées pour tester, à climat constant, comment l'urbanisation et les différents modes de gestion au cours du temps peuvent impacter la réponse hydrologique.

\section{REMERCIEMENTS}

Les données proviennent du projet AVuPUR (contrat ANR-07-VULN-01), de l'OTHU, du Grand Lyon et du SIAHVY. Ce travail fait partie du projet EC2CO BVPU/ ROSENHY.

\section{REFERENCES}

Andrieu H. Et Chocat B. (2004) - Introduction to the special issue on urban hydrology. J. Hydrol. 299(3-4) 163-165

BACH, M AND Ostrowski M. (2013) - Analysis of intensively used catchments based on integrated modelling. Journal of Hydrology. 485 148-161

Biegel M., Schanze J. And Krebs P. (2005) - ArcEGMO-urban - hydrological model for point sources in river basins. Water Science \& Technology. 52(5) 249-256

Branger F., Kermadi S., Jacqueminet C., Michel K., Labbas M. (2013) - Assessment of the influence of land use data on the water balance components of a peri-urban catchment using a distributed modelling approach. J. Hydrol. 505 312-325

Braud I. ET Al. (2010) - The AVuPUR project (Assessing the Vulnerabiliy of Peri-Urbans Rivers): experimental setup, modelling strategy and first results. Proceedings of the 7th Novatech 2010 Conference, Lyon, France. 10p

Braud I., Breil P., Thollet F., Lagouy M., Branger F., JACQueminet C., Kermadi S., Michel K. (2013) - Evidence of the impact of urbanization on the hydrological regime of a 
medium-sized peri-urban catchment in France. J. Hydrol. 485 $5-23$

Braud I., Fletcher T.D., Andrieu H. (2013) - Hydrology of peri-urban catchments: Processes and modelling. J. Hydrol. 485 1-4

Dhi Danish Hydraulic Institue (2011) - Mike urban - building a simple Mouse model in MIKE URBAN. Technical report. DHI Water and Environment, Horsholm, Denmark

FAO (1998) - Crop Evaporation - Guidelines for computing crop water requirements. Irrigation and Drainage, FAO, Rome. 56

FLÜGEL W.-A. (1995) — Delineating hydrological response units by geographical information system analyses for regional hydrological modelling using PRMS/MMS in the drainage basin of the River Bröl, Germany. Hydrol. Process. 9 (3-4) 423-436

Furusho C., Chancibault K., Andrieu H. (2013) - Adapting the coupled hydrological model ISBA-TOPMODEL to the long-term hydrological cycles of suburban rivers: Evaluation and sensitivity analysis. J. Hydrol. 485 139-147

Gustafsson L., Winberg S., RefsgaArd A. (1996) - Towards a physically based model description of the urban aquatic environment. In Verworn, F.-R., editor. 7th ICUDSD, Hannover. $1467-1472$

INSAVALOR-SOGREAH (1997) - CANOE- Logiciel d'hydrologie urbaine. Conception et évaluation de réseaux d'assainissement. Simulation des pluies, des écoulements et de la qualité des eaux

Jacqueminet C., Kermadi S., Michel K., Beal D., Gagnage M., Branger F., JANKowfSKy S., BRAUd I. (2013) - Land cover mapping using aerial and VHR satellite images for distributed hydrological modeling of peri-urban catchments: application to the Yzeron catchment. J. Hydrol. 485 68-83

Jankowfsky S., Branger F., Braud I., Rodriguez F., Debionne S., Viallet P. (2014) - Assessing anthropogenic influence on the hydrology of small peri-urban catchments: development of the object-oriented PUMMA model by integrating urban and rural hydrological models. J. Hydrol., in revision
Kralisch S., Krause P., Fink M., Fischer C., Flügel W. (2007) - Component based environmental modelling using the JAMS framework. In: Oxley, L., Kulasiri, D. (Eds.). Proceedings of the MODSIM 2007 International Congress on Modelling and Simulation. Christchurch, New-Zealand. 812-818

Krause P., Base F., Bende U., Fink M., Flugel W. Et Pfennig B. (2006) - Multiscale investigations in a mesoscale catchment - hydrological modelling in the gGera catchment. Adv. Geosci. $953-61$

Labbas M., Branger F., Braud I., Kralisch S., Jacqueminet C., Kermadi S., Michel K., Joliveau T., Dodane C., Volte E. (2013) - Multi-scale approach to assess the impacts of land use evolution and rainwater management practices on the hydrology of periurban catchments: application to the Yzeron catchment $\left(150 \mathrm{~km}^{2}\right)$. Novatech 2013, Lyon, France. 10p

Le Coz J., Renard B., Bonnifait L., Branger F., Le Boursicaud R. (2013) - Combining hydraulic knowledge and uncertain gaugings in the estimation of hydrometric rating curves: a Bayesian approach. J. Hydrol. 509 573-587

Lhomme J., Bouvier C., Perrin J.L. (2004) - Applying a GIS-based geomorphological routing model in urban catchments. J. Hydrol. 299 (3-4) 203-216

Masson V., Champeaux J., Chauvin F., Meriguet C., Lacaze R. (2003) - A global database of land surface parameters at $1-\mathrm{km}$ resolution in meteorological and climate models. J. Clim. 16 1261-1282

Rodriguez F., Andrieu H. Et Morena F. (2008) - A distributed hydrological model for urbanized areas - model development and application to case studies. J. Hydrol. 351(3-4) 268-287

Schmitt L., Valette L., Valin K., Piegay H., Hallot E. (2004) - Proposition d'une méthode de typologie hydro-géomorphologique des cours d'eau et test sur un sous-bassin du Rhône (bassin de 1'Yzeron). Mosella. XXIX(3-4) 323-340

Vidal J.P., Martin E., Franchisteguy L., Baillon M., Soubeyroux J. (2010) - A 50-year high-resolution atmospheric reanalysis over France with the SAFRAN system. Int. J. Climatol. 30 1627-1644 